\title{
The Culture of Giving Feedback in Health Professional Education: Reflection of Medical Educators on Effective Feedback Behavior
}

\author{
Shrestha, $R .{ }^{1}$, Sethi, S. ${ }^{2}$, Kaur, $D .^{3}$, Kingsley, PA. ${ }^{4}$, Chavda, P.D. ${ }^{5}$, Moudgil, ${ }^{6}{ }^{6}$, Sharma, M. ${ }^{4}$
}

\begin{abstract}
Background: Despite the fact that feedback improves learner performance, the feedback culture remains primitive. This paper explores the feedback behavior from a medical educator perspective. Furthermore, we explored the specific areas of improvement and attempts to suggest solutions to develop an effective feedback culture.
\end{abstract}

Methods: The personal experience and opinion of 33 medical educators from 20 medical institutions of India and one each from Nepal and Sudan on feedback were analyzed with mixed quantitative and qualitative methods. The response to an online self-assessment form and the individual narratives during online discussion were analyzed. Finally, their suggestions on way forward to implementation of an effective feedback culture was summarized.

Results: On average $78 \%$ of the participants reported having followed the action mentioned in the checklist. Vague terms for giving feedback were used by 18 (54.5\%), and only 15 (45.5\%) planned for a specific time frame for student's reassessment. During deductive thematic analysis, the themes "preparation before feedback", "elicitation of feedback from the learner", "feedback delivery", "improvement plan after feedback", "application of feedback", and "review of feedback" were expressed. The proposed strategies for the implementation of effective feedback are summarized under the main headings of to whom, what, where, when, and how.

Conclusion: Results emphasize that a culture of continuous and effective feedback should be nurtured in medical education by training the faculties to deliver effective feedback and sensitizing the learners to self-reflect and receive the feedback as it is critical to the achievement of the predefined competency.

Keywords: competency-based health professional education, faculty development program, medical education, peer feedback, self-assessment, reflection

\section{Introduction}

Feedback is an integral part of a competencybased health professional's education (CBHPE) that enhances student performance which ultimately affects patient care, however, the quality of the feedback is crucial (Shepard, 2009). Feedback is an informed, nonevaluative, and objective appraisal of performance in which learners seek to find out more about the similarities and differences between their performance and the target performance (Ende, 1983; Hattie \& Timperley, 2007).

DOI: http://doi.org/10.4038/seajme.v15i2.353
${ }^{1}$ Dhulikhel Hospital-Kathmandu University Hospital, Kavre, Nepal

2 Pt. B.D.Sharma PGIMS, Rohtak, India

${ }^{3}$ All India Institute of Medical Sciences, Rishikesh, Uttarakhand, India

${ }^{4}$ Christian Medical College, Ludhiana, India

${ }^{5}$ GMERS Medical College, Gotri, Vadodara, India

${ }^{6}$ Punjab institute of medical sciences, Jalandhar, Punjab, India

Corresponding author: Dr Roshana Shrestha roshanashrestha@gmail.com 
However, feedback given at the wrong time, in the wrong way, with wrong content is not only highly ineffective but can also be damaging (Komegay et al., 2017). The feedback process should be timely, interactive, personalized, non-judgmental, and specific. It should be accompanied by explanation; with the integration of the recipient's perspectives, foster self-assessment, and reflection, and facilitate subsequent learner performance (Dawson et al., 2019).

Despite the widely known fact that effective feedback improves learning outcomes and performances in the future, the feedback culture is suboptimal (Boud \& Molloy, 2013a). The problem is graver in developing countries (Kulkarni et al., 2019). The available literature on feedback suggests that both faculties and students have different perceptions of feedback (Perera et al., 2008) and reveals dissatisfaction from both aspects (Adcroft, 2011; Ramani et al., 2017). Feedback provision introduced by the Indian medical council and some Nepali institutions is certainly a step in the right direction, however, the provision of feedback alone does not ensure its use (Aggarwal et al., 2016).

The balance of various behaviors of the educator is asserted to create the pathway to effective feedback (Johnson et al., 2016). Numerous models have been offered for providing effective feedback (Boud \& Molloy, 2013b; Jug et al., 2019; Bhattarai, 2007). In this study, we chose to target the educator's behavior and reflection in their medical education practice. The aim was to explore the current personal practices of giving feedback by medical educators. We not only performed an in-depth evaluation of their self-reflection on specific areas of improvement for providing effective feedback in the future but also summarized the suggested solutions and methods for the development of an effective feedback culture.

\section{Methods}

Study Design:

Mixed qualitative and quantitative

\section{Study setting/participants}

The study sample consisted of 33 medical educators from 20 different medical institutions, mainly from India and one each from Nepal, and Sudan. All participants were enrolled in a medical education fellowship program conducted by the Foundation for Advancement of International Medical Education and Research (FAIMER). As a part of an intersession discussion, they participated in an online interaction on a listserv called the Mentoring-Learning Web session. The ethical clearance was not required. This paper summarizes the online discussions which occurred from 18th-26th July 2020.

\section{Data sources/variables}

The data in this study stems from 3 different stages of online discussions: stage 1: An online self-assessment form with 21-items with a dichotomous response (Yes/No) which was based on a previously validated tool (John Hopkins University Faculty Development Program) based on previous publications (Ende, 1983; Katz, 1995). It was modified and validated by the moderation team (peer fellows, $\mathrm{n}=4$ and faculties, $\mathrm{n}=3$ ) (supplemental file 1). During stage 2 data was collected through the online discussion, where the participants elaborated on the specific items needed for further enhancement of effective feedback. In stage 3 , the way forward on how to implement the feedback in CBHPE was brainstormed. These narratives were used as data sources for qualitative analysis. The identity of the fellows was kept anonymous.

\section{Data analysis}

The categorical variables from outputs of stage 1 were expressed as frequency/proportion. The individual narratives from stages 2 and 3 were compiled after removing the identifying information. The compiled document was read multiple times, coded by two authors (RS and SS) independently, and themes were generated with deductive methods based on predefined elements of effective feedback. 


\section{Results}

A total of 33 medical educators (8 males, 25 females) participated in the online forum. Among them 19 were clinical, 11 were pre/paraclinical, and 3 were dental medical educators. The summary of the result of stage
1 of the total 33 responses is depicted in figure 1. On average $78 \%$ of the participants responded positively to the actions. Eighteen $(55 \%)$ of the participants used vague terms like "good", "excellent", and "that was great" and 15 $(46 \%)$ specified a time frame for reassessment.
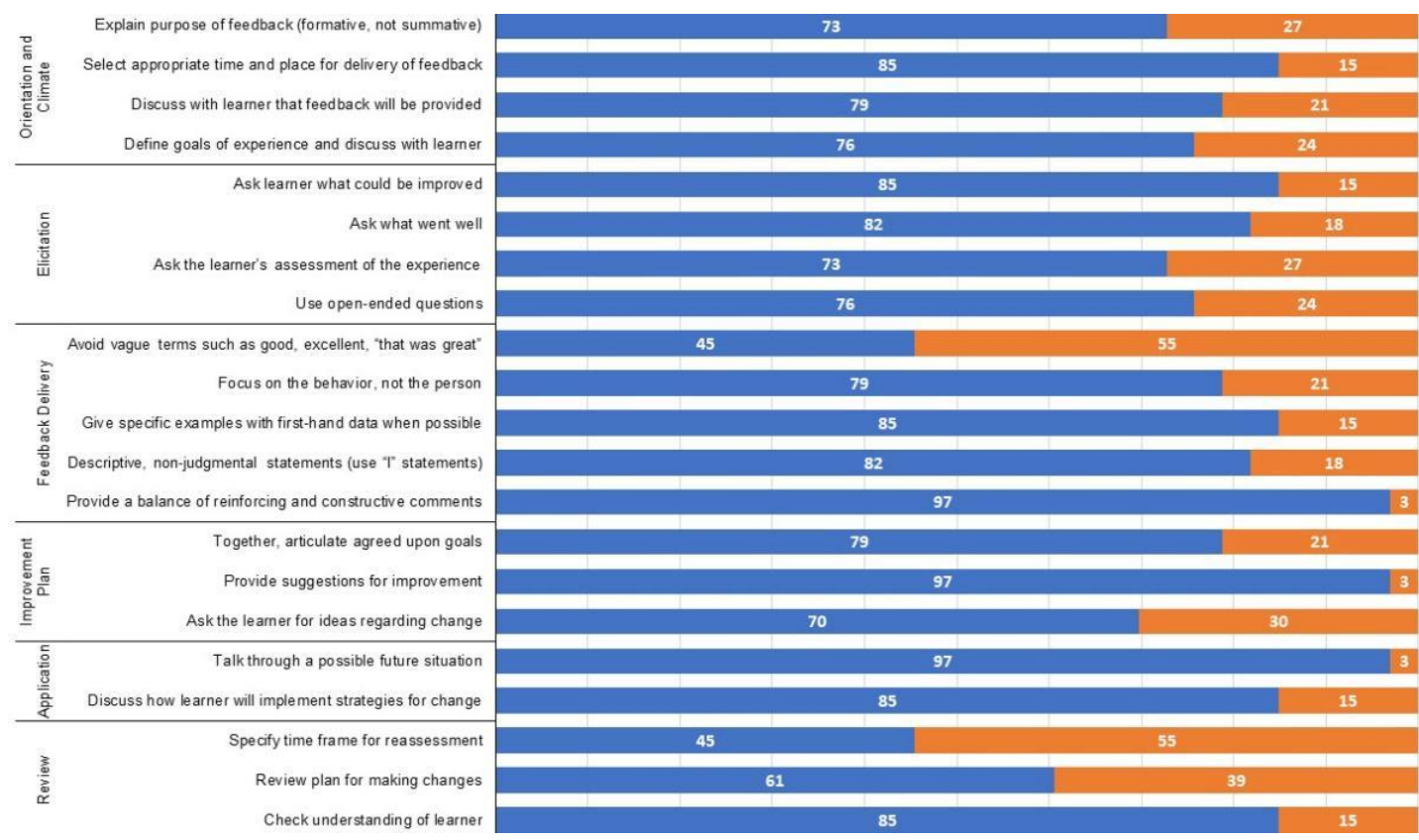

Figure 1. Self-assessment of the recent feedback by the fellows ( $n=33)$

In stage 2, fellows further elaborated on any two items that would further enhanced their technique of effective feedback in the future. The coding of individual responses was done and the six major themes generated are represented in Table 1.

The themes discussed were "preparation before feedback", "elicitation of feedback from the learner", "feedback delivery", "improvement plan after feedback", "application of feedback", and "review of feedback".

\section{Preparation before feedback}

The first common theme discussed by the 14 fellows (42.4\%) was the need for better preparation before feedback. They expressed the need for a definite goal: "needs to have welldefined goals, or else we don't justify it and the whole purpose of the feedback is defeated." Pre-Information and selection of appropriate time and place for the feedback delivery was thought to be important by a few $(n=8)$ of them: "I have been giving feedback more often in an impromptu manner, neither preparing myself not the student." During preparation the fellows also expressed their concerns about the need of explaining the purpose of feedback, "I found that it is crucial to mention that the feedback is a part of the formative assessment as it is meant to improve upon the performance of the student with time and this is not mere giving scores or marks to the student."

\section{Elicitation of feedback from the learner}

The need for improvement in the elicitation of feedback from the learner was apprehended by $23(69.7 \%)$ participants. They made explicit statements about the use of open-ended questions when giving feedback: "With openended questions, I have come to the realization that it opens up all links for effective communication initiating a better response and greater commitment from the student". Asking 
the learner's opinion on the experience was thought to be important as well. Some of them wrote: "Many times, I took it for granted that provision of feedback was the onus of the faculty and ended up giving my views rather than opening the session by asking the learner to self-assess and reflect on their experience". Starting the dialogue by asking the learner " What went well" and then "what could be improved" was expressed to be vital but often missed during feedback: "I hardly give time for self-assessment, which molds them to respond to the feedback better, makes them self-aware of the weak areas and also helps the learner to disclose the hidden areas to the feedback provider", "This, in fact, will make the learner a stakeholder in his/her improvement as well as instill confidence in the learner and a zeal to learn."

Table 1. Details of items requiring further improvement for effective feedback in future

\begin{tabular}{llr}
\hline Theme & Codes & $\begin{array}{r}\text { Fellows } \\
\mathbf{n}(\%)\end{array}$ \\
\hline $\begin{array}{l}\text { Improvement of preparation } \\
\text { before feedback }\end{array}$ & Definite goals & $2(6)$ \\
& Pre-information that feedback will be provided & $3(9)$ \\
& $\begin{array}{l}\text { Selection of appropriate time and place for the feedback } \\
\text { delivery }\end{array}$ & $5(15)$ \\
& Explain the purpose of the feedback & $4(12)$ \\
\hline Improvement on elicitation of & Use of open-ended questions & $6(18)$ \\
feedback from the learner & Ask for learners view on the experience & $7(21)$ \\
& Ask the learner "what went well" & $2(6)$ \\
& Ask learner what could be improved & $6(18)$ \\
\hline Feedback Delivery & Balance of reinforcing and constructive comments (just right & $3(9)$ \\
& amount, too much praise) & $3(9)$ \\
& Use non-judgmental statements with "I" & $8(24)$ \\
& Focus on the behaviour, not the person & $5(15)$ \\
\hline Avoid vague terms such as good, excellent, "that was great & $2(6)$ \\
\hline Review & Ask the learner for ideas regarding change & $1(3)$ \\
\hline & Together, articulate agreed-upon goals & $1(3)$ \\
& Discuss how the learner will implement strategies for change & $5(15)$ \\
& Check the understanding of the learner & $7(21)$ \\
& Review plan for making changes & $15(46)$ \\
\hline & Specify the time frame for reassessment &
\end{tabular}

Feedback delivery

The concern about the balance of reinforcing and constructive comments were expressed. They thought that the content should be of the right amount and not too much buttering with praise: "I need to focus on the issue and not encumber them with all the ideas and suggestions", "Praising can, at times, dilute the purpose of giving feedback with the receiver being falsely confident of his performance". They pointed out that addressing feedback statements with "l" would make the feedback non-judgmental, "There is little heat when it comes from I". A common recurring theme by 8 participants (24\%) was the need to focus on the behavior, not the person. Some of the interesting verbatim reflecting the theme were: "I learned to dissociate a behavior from the person and NOT make an impression of the student based on behavior", "I realize that this feedback delivery of not blaming the individual would give a lot of confidence and reassurance to the student and sustain his enthusiasm and drive". Avoidance of vague terms when giving feedback was highlighted: "Although it is hard to check one's emotions and not to make such remarks, I will try to correct myself and not use these vague terms." 
Improvement plan and application of feedback

Critical comments were made on the practice gap that we seldom ask the learner for ideas regarding change and then articulate agreedupon goals: "probably driven by overenthusiasm, I just realized that most of the time I am bombarding them with my unidirectional ideas of the perfect strategy for improvement without their involvement", "It is very essential to wrap-up and to ask the receiver to summarize and to make sure that both of you are on the same page and at the same line". Moreover, to apply the feedback for further improvement in the future performances, a participant stated that discussion on how the learner will implement strategies for change is pivotal: "In addition to formulating the action plan for learning, the strategies to implement needs to be described"

\section{Review}

Most of the participants 27 (81.8\%) formulated comments on reviewing the feedback before the closure. The necessity of checking for understanding of the student and review plan for making changes was expressed: "Checking the understanding of the learner and reviewing the plan for improvements is important.", "We also assume that our messages and suggestions are clear and considered by our students, but that may not be true," "I need to revise my working and make sure that implementation of the plan also gets assessed". A total of 15 participants (45.5\%) referred explicitly to the gap of not specifying the time frame for reassessment: "Not discussing this point will leave the feedback receiver clueless and casual about the need for re-visiting the same chapter/concept/task for future improvement." The importance to complete the feedback loop was echoed.

Reflecting on their current practice and extensive in-depth discussion, in stage 3 , the main principles of implementation of the effective feedback in CBHPE was brainstormed (Table 2)

Table 2. Suggestions for implementation of feedback in CBHPE

\begin{tabular}{|c|c|}
\hline $\begin{array}{l}\text { To } \\
\text { whom }\end{array}$ & $\begin{array}{ll}\text { - } & \text { Self (self-reflection) } \\
\text { - } & \text { Students: Undergraduate, interns, postgraduate, } \\
\text { - } & \text { Peers (student to student, teacher to teacher) }\end{array}$ \\
\hline What & $\begin{array}{ll}\text { - } & \text { A two-way dialogue } \\
\text { - } & \text { Learning goal-oriented (technical and nontechnical competencies) } \\
\text { - } & \text { Self-regulatory including cognitive, metacognitive, and motivational factors needed for the performance } \\
\text { - } & \text { Reflecting on the specific task / direct observation/ performance } \\
\text { - } & \text { Constructive, non-judgmental comments } \\
\text { - } & \text { Can be formal (template, checklists) vs informal } \\
\text { - } & \text { Individual feedback vs group feedback } \\
\text { - } & \text { Balanced feedback (positive/negative) }\end{array}$ \\
\hline When & 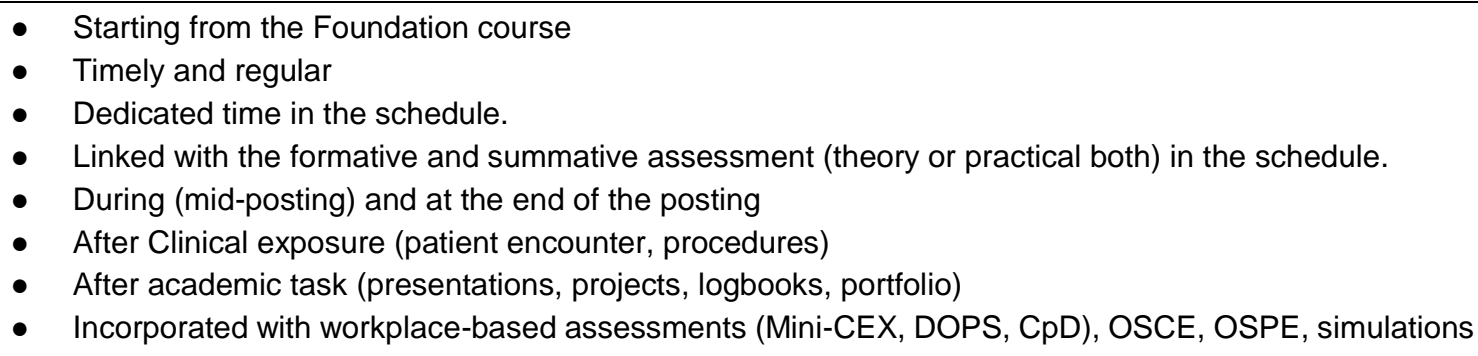 \\
\hline How & $\begin{array}{l}\text { - } \quad \text { By the establishment of a safe and positive learning environment } \\
\text { - } \quad \text { By clarifying the goals, expectations, and purpose of the feedback session before starting } \\
\text { - } \quad \text { By facilitating the learners to speak up with open-ended questions in respectful tone } \\
\text { - } \quad \text { By active listening (listening without interrupting, using reflective verbal and nonverbal cues) }\end{array}$ \\
\hline
\end{tabular}




\begin{tabular}{|c|c|}
\hline & $\begin{array}{l}\text { - By multiple techniques according to settings and need. "One size doesn't fit all" (Pendleton, Sandwich, } \\
\text { - } \quad \text {-step micro skill, Ask-Tell-Ask) } \\
\text { together". } \\
\text { - By respecting learners' autonomy and empowering the learner to formulate the agreed-upon action } \\
\text { - } \quad \text { Blan for improvement } \\
\text { - By encollaboratively formulating the action plan for follow-up and re-assessing progress with the learners } \\
\text { By feedback-seeking behavior }\end{array}$ \\
\hline Where & $\begin{array}{l}\text { - Can be anywhere but in a safe and private environment: clinics, faculty room, operation room lounge } \\
\text { Bedside clinics, academic rooms, community field visits. Considering COVID -19 situation online } \\
\text { feedback session (via Google form or synchronous virtual meeting) was suggested }\end{array}$ \\
\hline
\end{tabular}

\section{Discussion}

Our study provides several insights into the medical educator's perspectives on key issues that influence the effectiveness of the feedback given to the learner. The self-reflection of the 33 participants on their feedback practice revealed that $78 \%$ of the participants reported having followed the action mentioned in the checklist. Deductive thematic analysis revealed themes for future improvements and the proposed strategies for the implementation of effective feedback were summarized under the main headings of to whom, what, where, when, and how.

Over the past few years, the culture of giving feedback has been revolutionized worldwide. Despite its proven benefit in learning and abundance of proposed models (Bernard et al., 2011), the importance of feedback is still not fully appreciated and publications have highlighted the concern about its appropriate use in the developing world (Kulkarni et al., 2019; Shrivastava et al., 2014; Lüdeke et al., 2020; Adloki, 2020). Fellows mentioned that giving feedback should be started with selfreflection, it can be implemented to any learners at any stage of medical education and should be practiced with peers (students to students and teachers to teachers). They expressed that, "one size doesn't fit all", and suggested that a suitable one should be used from the multiple available techniques according to the settings and need (Jug et al., 2019). The main goal is to enable the learner to reflect on their experience in order to facilitate future improvements and experience deep learning (Ende, 1983). The fellows thought that the culture of feedback should be ingrained from the early stage of medical education, with a dedicated time allocated in the schedule Additionally, it was felt by the participants that effective feedback should be linked with formative as well as summative assessment after any academic task or clinical exposure with workplace-based assessments.

\section{Preparation before feedback}

Conducting feedback sessions without preparation may lead to disastrous outcomes (Hattie and Timperley, 2007). Providing a physically comfortable learning environment with appropriate seating arrangements in a private and safe atmosphere with minimal distractions was thought to be important to fully engage in the feedback process (Hulse and Robert, 2014). Delva et al. mentioned in their study that the learners should explicitly know that the feedback is for the individual developments, not for the learner's evaluation (2013). It is also important to pre-inform the learners about the purpose, time and place of providing. Taking the learner by surprise with the feedback conversation was not encouraged by the fellows. Thus, establishing a safe and engaging learning environment was one of the critical points mentioned by the fellows. They expressed that it would be helpful if the learner knows details ahead of time, including the setting; learner and instructor roles; goals and expectations.

\section{Elicitation of feedback from the learner (Self- reflection)}

The need for engaging the learner actively in the feedback conversation, encouraging the deliberate self-reflective behavior was echoed 
by the fellows "reflection and feedback should be carried out together". With the recent paradigm shift towards CBHPE, the importance of self-assessment is becoming more crucial (Eva and Regehr, 2008). Studies have proved that learners generate the majority of learning goals from their own self-assessment and that the learning goals stem from a combination of self-assessment and feedback from the teacher (Hattie and Timperley, 2007; Bounds et al., 2013). Strategies that encourage and demonstrate confidence in the learner's ability to reflect on their own experience, like using open-ended questions, listening without interrupting, using reflective verbal and nonverbal cues should be practiced. As they nurture the reflective practice, they start to compare their performance to the target performance, thereby it offers an opportunity for them to clarify their performance gap (Boud and Molloy, 2013b). The participants accepted that medical educators, usually display an authoritarian attitude and ask questions, interrupt, make assumptions, and jump to teach concepts without jointly identifying the causes for the particular action. Studies have shown that learners may not implement the advice given during the feedback particularly when there was a mismatch between selfperceptions and teachers' perspectives (Lockyer et al., 2003).

Effective and ineffective behavior during feedback delivery.

Providing balanced feedback, including positive and negative comments can be challenging. In the event that the performance was good or excellent, the instructor should reinforce the action and its attributes so that it can be continued in the future. However, the fellows also raised the concern that the feedback skewed towards positive with sugar-coating can be ineffective and may fail to target the actionable areas needing improvement. Maintaining the focus of feedback on the learner's behaviors and performance rather than personal attributes is of utmost importance (Feedback fundamentals, 2015). The fellows acknowledged that they used vague terms "good, excellent, great" or strong adjectives "never, always" and committed that they would avoid them in the future feedback sessions. Comparing the learner's performance to that of other learners rather than to the standard of performance can be detrimental. The observed concrete performance should be explicitly compared to the standard of performance. The participants recommended providing feedback in the first person, using "l" statements rather than "you" statements.

\section{Mutual articulation of the improvement plan and application of feedback.}

Engaging in a two-way dialogue with the active engagement of the learner to articulate the improvement plans were expressed by the participants. Allowing for a conversation that includes the learner's views on the way forward should be mutually discussed and agreed upon (Gregory and Levy, 2015). When performance is sub-optimal, the teacher should actively seek the learner's idea on how to cover the performance gap. The fellows voiced that feedback needs to take a step further and empower the learner to formulate the agreedupon strategies to improve the currently observed performance to the desired performance.

The climax: Clarification, summarization and review plan after the feedback

Finally, some of the participant fellows discussed the need for an opportunity for learners to clarify understanding. Assessing the learner's perception and interpretation of feedback to ensure feedback was received, understood, and can be applied to practice needs to be done before the closure (Ramani and Krackov, 2012). Posing questions that ask the learner to think about the 'lessons learned' from the experience can be helpful to summarize. Specifying with the learner to follow-up and re-assess progress is important and needs to be listed in the implementation plan in Table 2.

\section{Limitations}

This was mixed type research with a small group of teachers selected from a fellowship program. The questionnaire reflected only one 
of the recent feedback delivered by the faculties. Their characteristics may be different compared to other educators hence cannot be generalized.

\section{Conclusions}

Feedback is a complex process with various components dependent on each other. A culture of continuous and effective feedback should be nurtured in medical education in our part of the world. With the recent country-wise implementation of CBHPE in India and the introduction of the concept in other developing countries, it is high time to train the faculties to deliver effective feedback. The students should also be sensitized and trained to self-reflect and receive feedback. Feedback culture should be embedded in the curriculum and should be an integral part of formative assessment in CBHPE.

\section{Acknowledgement}

All the fellows (2018, 2019 and 2020) participating in the fellowship program conducted by the Foundation for Advancement of International Medical Education and Research at Regional Institute at Christian Medical College, Ludhiana (CMCL) in India. Gratitude to Professor Dr. Dinesh Badyal and other faculties for their kind support and inspirations.

\section{Conflict of interest: none}

Criteria for inclusion in the authors' contributors' list

The manuscript has been read and approved by all the authors, that the requirements for authorship as stated earlier in this document have been met, and that each author believes that the manuscript represents honest work, if that information is not provided in another form

\section{References}

Adcroft, A., 2011. 'The mythology of feedback.' Higher Education Research \& Development, 30,4 , pp. 405-419.
Adkoli, B. Feedback culture: How do we develop it? [Internet]. 2020 [cited 2020 Dec 28]. Available from: https://timesofindia.indiatimes.com/blogs /bv-lens/feedback-culture-how-do-we-develop-it/

Aggarwal, M., Singh, S., Sharma, A., Singh, P., et al., 2016. 'Impact of structured verbal feedback module in medical education: A questionnaireand test score-based analysis'. Int J Appl Basic Med Res. 6, 3, pp. 220-225.

Bernard, A.W., Kman, N.E., Khandelwal, S., 2011. 'Feedback in the emergency medicine clerkship'. Western Journal of Emergency Medicine, 12, 4, pp. 537-542.

Bhattarai, M., 2007. 'ABCDEFG IS - the principle of constructive feedback'. JNMA J Nepal Med Assoc, 46, 167, pp. 151-156.

Boud, D. \& Molloy, E., 2013a, Feedback in higher and professional education: Understanding it and doing it well. Routledge, USA.

Boud, D., Molloy, E., 2013b. Rethinking models of feedback for learning: the challenge of design [Internet]. Vol. 38, Assessment \& Evaluation in Higher Education. pp. 698-712. Available at: http://dx.doi.org/10.1080/02602938.2012.69146 2

Bounds, R., Bush, C., Aghera, A., Rodriguez, N., Stansfield, R. B., Santen, S. A., \& MERC at CORD Feedback Study Group 2013. Emergency medicine residents' self-assessments play a critical role when receiving feedback. Acad Emerg Med. 20, 10, pp. 1055-1061.

Dawson $\mathrm{P}$, Henderson M, Mahoney P, Phillips M, Ryan T, Boud D \& Molloy E 2019. 'What makes for effective feedback: staff and student perspectives' Assessment \& Evaluation in Higher Education, 44, 1, pp. 25-36.

Delva, D., Sargeant, J., Miller, S., Holland, J., Alexiadis Brown, P., Leblanc, C., Lightfoot, K., \& Mann, K, 2013. 'Encouraging residents to seek feedback. Med Teach' [Online]. 35(12):e162531. Available from: http://dx.doi.org/10. 3109/0142159X.2013.806791Accessed on $16 / 07 / 2021$

Ende, J., 1983. 'Feedback in clinical medical education', JAMA: Journal of the American Medical Association, 250, 6, pp. 777-781. Available at: https://www.ncbi.nlm.nih.gov/ pubmed/6876333.

Eva, K.W., Regehr, G., 2008 "I'll never play professional football" and other fallacies of selfassessment'. J Contin Educ Health Prof. 28, 1, pp. 14-19.

Feedback Fundamentals: Specific, Timely, and Balanced [Internet]. Your First Leadership Job. 2015. pp. 173-180. Available from: http://dx.doi.org/10.1002/9781119153849.ch15 
Gregory, J. B., \& Levy, P. E., 2015. Feedback delivery and the role of the feedback provider. In J. B. Gregory \& P. E. Levy, Using feedback in organizational consulting (pp. 47-62). American Psychological https://doi.org/10.1037/14619-005

Hattie, J. and Timperley, H., 2007. 'The Power of Feedback', Review of Educational Research, pp. 81-112. doi: 10.3102/003465430298487.

Hulse, D., Robert, T., 2014. Preplanning for Feedback in Clinical Supervision: Enhancing Readiness for Feedback Exchange [Internet]. The Journal for Counselor Preparation and Supervision. 2014. Available from: http://dx.doi.org/10.7729/62.1091

John Hopkins University Faculty Development Program. Checklist for Small Group Practice Session [Internet]. [Cited 2020 Dec 23]. Available from: https://www.hopkinsmedicine.org/institute excellence_education/_documents/Feedback\% 2 01\%20Documents.pdf

Johnson, C., Keating, J., Boud, D., Dalton, M., Kiegaldie, D., Hay, M., McGrath, B., McKenzie, W. A., Nair, K. B., Nestel, D., Palermo, C., \& Molloy, E. K., 2016. Identifying educator behaviours for high quality verbal feedback in health professions education: literature review and expert refinement. BMC Med Educ, 16, 1, p. 96.

Jug, R., Jiang, X.S., Bean, S.M., 2019. 'Giving and Receiving Effective Feedback: A Review Article and How-To Guide. Arch Pathol Lab Med' [online]143(2):244-50. Available from: http://dx.doi.org/10.5858/arpa.2018-0058-RA

Katz, P. O., 1995. 'Providing feedback', Gastrointestinal endoscopy clinics of North America, 5, 2, pp. 347-355.
Kornegay, J. G., Kraut, A., Manthey D., Omron R., Caretta-Weyer, H., Kuhn, G., Martin, S., \& Yarris, L. M. 2017. 'Feedback in Medical Education: A Critical Appraisal'. AEM Educ Train. 22, 1,2, pp. 98-109.

Kulkarni, P., Pushpalatha, K., Bhat, D., 2019. 'Medical education in India: Past, present, and future. Vol. 7, APIK Journal of Internal Medicine. Vol.7: $69 . \quad$ Available from: http://dx.doi.org/10.4103/ajim.ajim_13_19. Accessed on 16/07/2021

Lüdeke, A. B. E. K., Lüdeke, A. B. E. and Olaya, J. F. G.,2020. Effective Feedback, An Essential Component of all Stages in Medical Education [Internet]. Vol. 61, Universitas Médica. Available from: http://dx.doi.org/10.11144/javeriana.umed 61-3.feed

Perera, J., Lee, N., Win, K., Perera, J., Wijesuriya, L., 2008. 'Formative feedback to students: the mismatch between faculty perceptions and student expectations'. Med Teach.30, 4. Pp. 395399.

Ramani, S., Krackov, S.K., 2012 Twelve tips for giving feedback effectively in the clinical environment. Med Teach. 34, 10, pp. 787-791.

Ramani, S., Post, SE., Könings, K., Mann K Katz, J. T., \& van der Vleuten, C, 2017.'It's Just Not the Culture": A Qualitative Study Exploring Residents' Perceptions of the Impact of Institutional Culture on Feedback '. Vol. 29, Teaching and Learning in Medicine. 2: 153-61.

Shepard, L. A., 2009. 'The Role of Assessment in a Learning Culture', Journal of Education, pp. 95106. doi: 10.1177/0022057409189001-207.

Shrivastava, S., Shrivastava, P., Ramasamy, J., 2014. 'Effective feedback: An indispensable tool for improvement in quality of medical education'. Journal of Pedagogic Development 4(1): 12-20. 